\title{
Endomorphisms of superelliptic jacobians
}

\author{
Yuri G. Zarhin
}

Published online: 31 May 2008

C) Springer-Verlag 2008

\section{Erratum to: Math. Z.}

\section{DOI:10.1007/s00209-008-0342-5}

Proof of Lemma 3.4 The non-zero element of $\operatorname{End}_{K}(X, i) \otimes \mathbb{Q}$ attached to $\mathcal{J}$ should be defined as $e_{\mathcal{J}}=\sum_{s \in \mathcal{J}} e_{s}$.

Proof of Theorem 3.5, second displayed formula. Change $\operatorname{Lie}_{K_{a}}(X)_{\kappa}$ to $\operatorname{Lie}_{K_{a}}(X)_{\tau}$.

Proof of Theorem 3.5, third displayed formula. Change $\sigma\left(\operatorname{Lie}_{K_{a}}\left(X_{S}\right)_{\tau}\right)$ to $\sigma\left(\operatorname{Lie}_{K_{a}}\left(X_{t}\right)_{\tau}\right)$.

The online version of the original article can be found under doi:10.1007/s00209-008-0342-5.

Yu. G. Zarhin $(\bowtie)$

Department of Mathematics, Pennsylvania State University, University Park, PA 16802, USA

e-mail: zarhin@math.psu.edu

Yu. G. Zarhin

Institute for Mathematical Problems in Biology, Russian Academy of Sciences,

Pushchino, Moscow, Russia 